STELLAR KNOWLEDGE TO AND FROM LIGHT ELEMENTS 


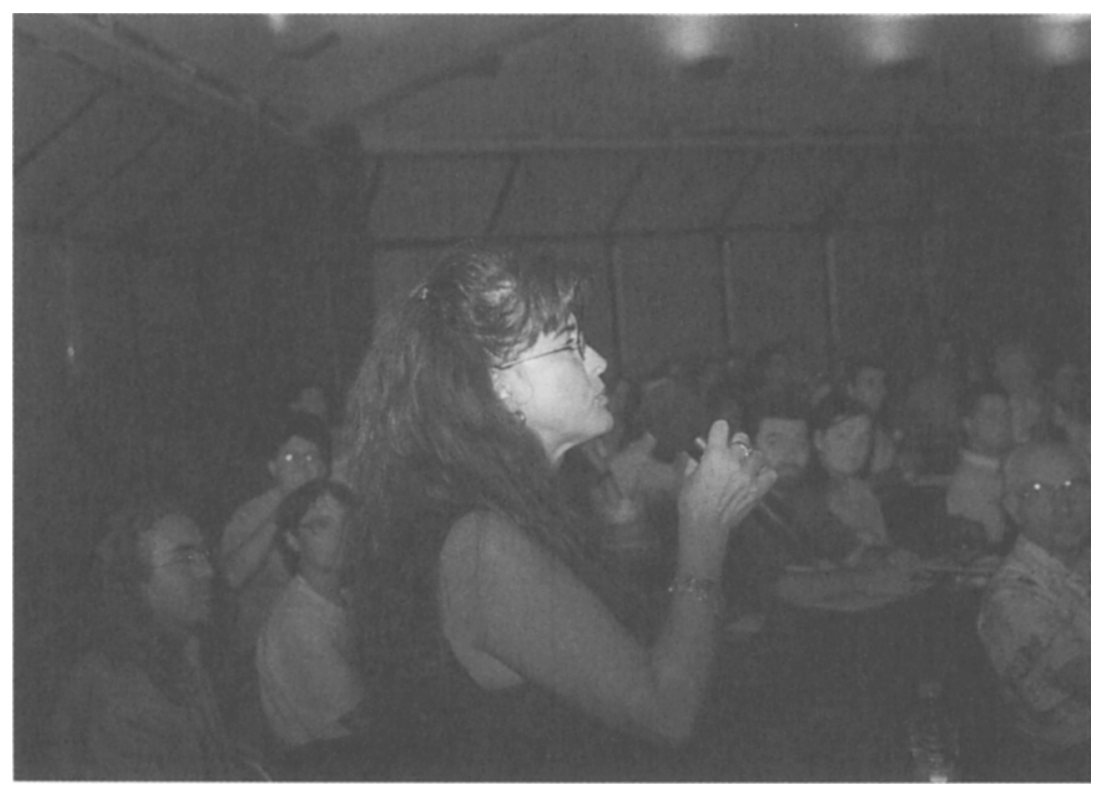

Francesca Matteucci, with Roger Cayrel, on the right, and Renan de Medeiros and Monique Spite, on the left 


\title{
Effects of Photospheric Temperature Inhomogeneities on Lithium abundance Determinations (2D)
}

\author{
Roger Cayrel \\ Observatoire de Paris, DASGAL, 61, Observatoire de Paris \\ F75014 Paris, France \\ Matthias Steffen \\ Astrophysikalisches Institut Potsdam, An der Sternwarte 16 \\ D-14482 Potsdam, Germany
}

\begin{abstract}
Based on detailed 2D radiation hydrodynamics (RHD) simulations, we have investigated the effects of photospheric temperature inhomogeneities induced by convection on spectroscopic determinations of the lithium abundance. Computations have been performed both for the solar case and for a metal-poor dwarf. NLTE effects are taken into account, using a five-level atomic model for Li I. Comparisons are presented with traditional 1D models having the same effective temperature and gravity. The net result is that, while LTE results differ dramatically between 1D and 2D models, especially in the metal-poor case, this does not remain true when NLTE effects are included: 1D/2D differences in the inferred NLTE Li abundance are always well below 0.1 dex. The present computations still assume LTE in the continuum. New computations removing this assumption are planned for the near future.
\end{abstract}

\section{Introduction}

It would be an offense to the audience here to pretend to explain why it is important to determine accurately the abundance of ${ }^{6} \mathrm{Li}$ and ${ }^{7} \mathrm{Li}$ in the oldest stars. In this respect, we have nothing to add to the exposition by François Spite (this volume). We will right away mention the two major problems which may cast doubts on our real knowledge of the actual initial abundance of $\mathrm{Li}$ in the oldest stars, and consequently in the primordial matter. (i) While standard models of the internal structure of metal-poor dwarfs do not deplete ${ }^{7} \mathrm{Li}$, more sophisticated models including rotationally induced mixing (Pinsonneault et al. 1992) have predicted that the measured abundance in the photosphere is 5 to 10 times less than the initial abundance representative of Big Bang material. (ii) On top of that, Kurucz (1995) claimed that the hot and cold convective structures produce large effects in metal-poor stellar photospheres, where the convection zone reaches the line formation layers. The claimed effect is an overionization of $\mathrm{Li}$ by a factor of 10 , leading to an underestimation of the abundance of $\mathrm{Li}$ when derived from the resonance line of $\operatorname{Li~I}(\lambda 670.8 \mathrm{~nm})$ in the usual way. 
If these two statements are correct, the true abundance of $\mathrm{Li}$ in primordial matter is 50 to 100 times higher than the value derived from 1D, LTE models of halo subdwarfs so far. The first factor of 5 to 10 has been discussed in a previous paper by Ryan (this symposium), and shown to be likely much smaller, of the order of 1 to 1.4. We shall not come back to this point, which we consider as very well treated.

Before this symposium, a single paper (Asplund et al. 1999) has dealt with the question of the other factor of 10 claimed by Kurucz (1995), whose arguments were based on a simplified two-column model. In contrast, the work by Asplund et al. relies on realistic 3D hydrodynamical models, similar to the simulations of the solar granulation (Stein \& Nordlund 1998), but with parameters appropriate for two metal-poor stars: HD 140283 and HD 84937, both subgiants. The computation of the lithium resonance line was made under the assumption of LTE, and the correction to be applied to the Li abundance derived from standard $1 \mathrm{D}$ models was found to be large, of the order of -0.2 to -0.35 dex. Note that these corrections have the opposite sign as Kurucz's prediction! However, Kiselman (1997, 1998) had shown, in the solar case, that NLTE and LTE computations lead to significantly different values of equivalent widths of the $\operatorname{Li}$ I $\lambda 670.8 \mathrm{~nm}$ line over hot and cold structures (see Fig. 3 of his 1997 paper, top panel).

For this reason, we decided to undertake NLTE radiation hydrodynamics computations for the case of a metal-poor star, and we report here on the results of this investigation. In the next section we recall former work related to simulations of the solar granulation, a useful benchmark for checking the theory, but not directly applicable to metal-poor stars. In section 3 we describe the assumptions underlying the construction of the 2D RHD models used for the spatially resolved computation of the lithium resonance line. Section 4 gives the description of the NLTE treatment of the Li atom, and section 5 summarizes our results and compares them to those presented by M. Asplund (this symposium). Finally, our conclusions are listed in section 6.

\section{Former work at solar metallicity}

While there is only one paper dealing with multidimensional atmospheres for metal-poor stars (cited above), there are several studies for the solar case, aimed at understanding the variation of the continuous radiation intensity (granulation), and the behavior of spectral lines across the solar granulation pattern. Several of these works use snapshots from 3D simulations by Stein \& Nordlund (1998), such as Kiselman $(1997,1998)$ and Uitenbroek (1998). Gadun \& Pavlenko (1997) use their own 2D simulations.

Of particular interest for us are the papers dealing with the combined effects of multidimensional structures and NLTE (Kiselman and Uitenbroek). It is clear from Kiselman (1997) that the NLTE behavior of the Li I $\lambda 670.8 \mathrm{~nm}$ resonance line is drastically different from its LTE behavior, in $2 \mathrm{D}$ as well as in $3 \mathrm{D}$ models. The result which is the most relevant for us is the difference of 30 per cent on the predicted mean equivalent width $\langle W\rangle$ of the line, leading to a similar change in the derived lithium abundance (NLTE/LTE abundance correction $+0.15 \mathrm{dex}$ ). But another interesting difference is the reverse behavior of the equivalent width $W$ as a function of surface continuum brightness $I_{c}$. While LTE computations 


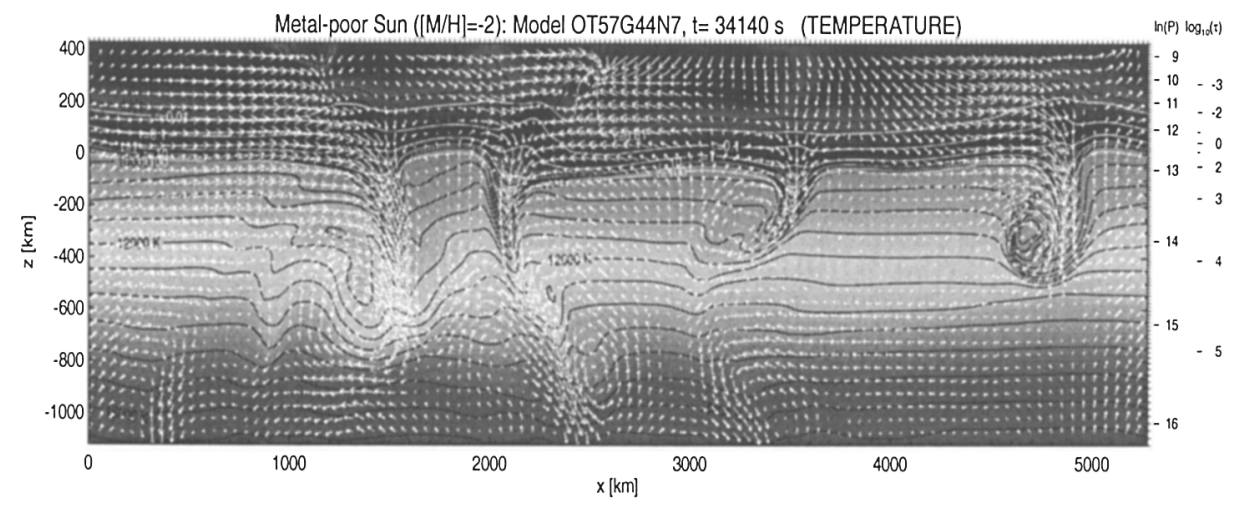

Figure 1. Snapshot from the metal-poor Sun simulation, showing the velocity field (arrows) and temperature structure (black contour lines). White lines are curves of iso-optical depth. The origin of the geometrical scale (left) corresponds to $\tau_{\text {Ross }}=1$; scales at right refer to average gas pressure $P$ and average optical depth $\tau_{\text {Ross. }}$. This simulation was done on a $210 \times 106$ grid (tick marks at right and top), with a vertical extent of about $1500 \mathrm{~km}\left(\approx 7 H_{p}\right)$, and a horizontal period of $5250 \mathrm{~km}$ (upper and lower boundaries 'open', lateral boundaries periodic).

result in a strongly positive slope in the $W$ versus $I_{c}$ diagram (with a large scatter around the mean relation), NLTE computations show a slightly negative slope and a much tighter (anti-)correlation between $I_{c}$ and $W$. This reflects the fact that the population of the Li I levels is much more controlled by the local temperature in LTE than in NLTE, where, for weak lines, the photoionization rates play the dominant role. So, even if the general conclusion of the above mentioned papers is that, in the solar case, the abundance determination of $\mathrm{Li}$ is not strongly affected by the combined effects of temperature fluctuations and NLTE, in comparison to what is obtained with classical 1D models having the same effective temperature and gravity, it appears unsafe to compute abundances from multidimensional stellar atmospheres based on the assumption of LTE line formation.

\section{2D radiation hydrodynamics models}

Our LTE and NLTE computations have been performed on the basis of several snapshots from a 2D numerical simulation of convection in a stellar envelope having the same effective temperature and gravity as the Sun, but a 100 times smaller metallicity. Basically, the time dependent equations of hydrodynamics are solved for a compressible fluid, with an energy equation including 3 terms: turbulent and shock dissipation of kinetic energy, diffusive transport of heat, and radiative energy exchange. The main limitation of the code is the restriction of the flow to two spatial dimensions. Magnetic fields and rotation are ignored.

Apart from these simplifications, as much realistic physics as possible is included. The equation of state accounts for ionization of hydrogen and helium 
as well as $\mathrm{H}_{2}$ molecule formation, opacities have been adapted from Kurucz's ATLAS code and include line absorption. For the computation of the radiative energy balance, we employ a multi-dimensional, non-local, frequency-dependent radiative transfer scheme, actually solving the transfer equation along 26880 independent rays of various inclinations, using an efficient modified Feautrier method (Feautrier 1964). At the bottom boundary, inflowing matter has a given specific entropy, which is adjusted to produce the prescribed effective temperature of the atmosphere. Energy dissipation on small scales is roughly modeled by introducing a subgrid scale eddy viscosity, depending on the grid resolution and local velocity gradients in the usual way. Details of the employed hydrodynamics code can be found in Ludwig et al. (1994) and Freytag et al. (1996). Fig. 1 shows a sample snapshot from our metal-poor Sun simulation. Note the complex velocity pattern and the occurrence of very strong temperature gradients. The relevant region for the formation of the $\mathrm{Li} \mathrm{I}$ line is the $\tau=0.1$ contour line.

\section{NLTE computation of the $\mathrm{Li}$ I resonance line}

The computation of the Li I spectrum is greatly simplified by the fact that all lines of $\mathrm{Li} \mathrm{I}$ are weak, $\mathrm{Li}$ being a trace element. So the radiation field in the line is, to first approximation, the same as the continuous radiation field, a single iteration being sufficient for taking care of the small perturbation of the monochromatic radiation field brought about by the line.

We have, as a first step, approximated the Li I atomic configuration by a five level atom, exactly as done by Uitenbroek (1998). This leaves six permitted bound-bound transitions, and five photoionization rates needing the computation of the continuous radiation field at frequencies above the threshold, until the contribution of the product of photoionization cross-section and mean intensity of the radiation becomes negligible. Because in the UV the contribution of lines to the opacity is important, we have used Kurucz's Opacity Distribution Functions (ATLAS 9) for the relevant metallicity. This multiplies the computation time by 12 , as each opacity bin is subdivided into 12 subintervals. So, for each snapshot, the transfer equations must be solved for about $12 \times 120$ wavelengths, along 26880 different rays (note that these extensive computations are done only after the actual hydrodynamical simulation for a few selected snapshots). After this, it is possible to compute all the coefficients in the equations of statistical equilibrium (see Mihalas 1970, p. 144). Once the departure coefficients $b_{i}$ are evaluated, the line can be computed using the source function:

$$
S=\frac{\kappa_{c}}{\kappa_{l}+\kappa_{c}} B_{\nu}+\frac{\kappa_{l}}{\kappa_{l}+\kappa_{c}} S_{l}
$$

where:

$$
S_{l}=S_{i j}=\frac{2 h \nu_{i j}^{3}}{c^{2}} \frac{1}{\left(b_{i} / b_{j}\right) \exp \left(h \nu_{i j} / k T\right)-1}
$$

is the line source function. The departure coefficient for the lower level is $b_{i}$ and the one for the upper level $b_{j}$. The other notations are standard. Subscript "c" stands for continuum, and "l" for "line". Note that in NLTE the expression of the partition function is modified and becomes: 
DEPARTURE COEFFICIENTS

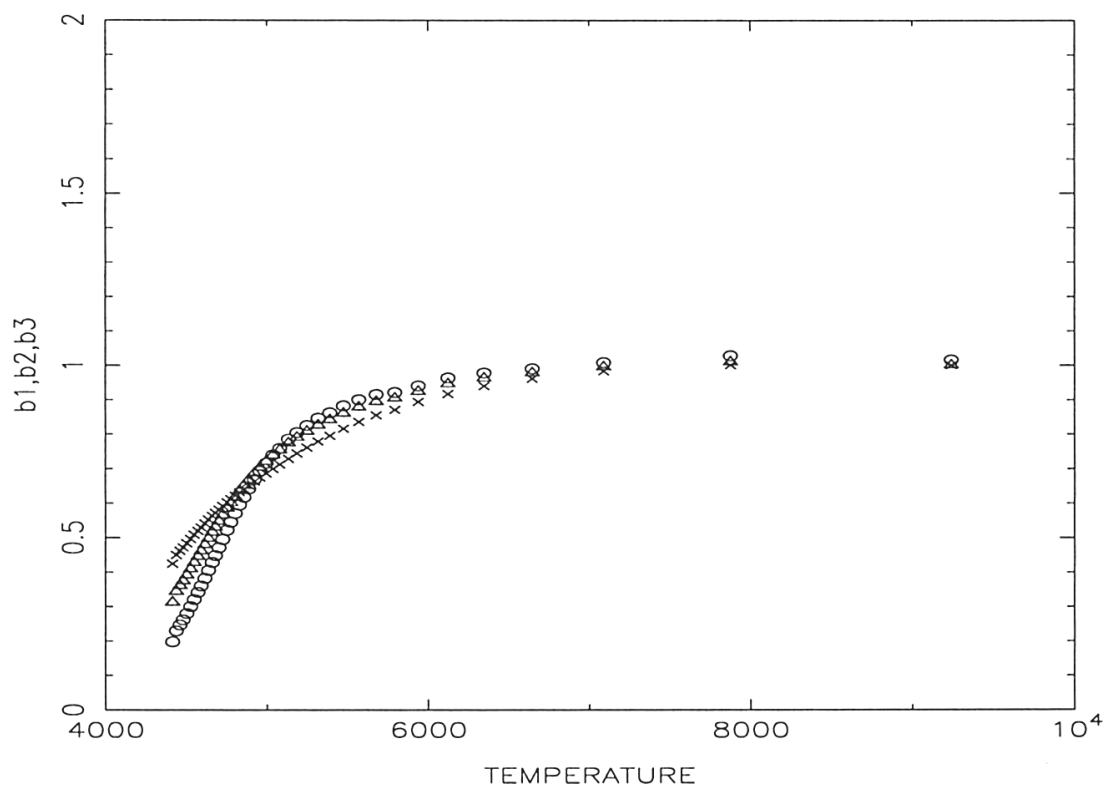

Figure 2. Variation of the first three departure coefficients with depth (actually temperature in this graph), based on an 1D ATLAS 9 model of the solar atmosphere (mixing-length parameter $\alpha=0.5$ ). Circles refer to the ground level $\left(b_{1}\right)$, triangles to the $2 \mathrm{p}$ level $\left(b_{2}\right)$, and crosses to the $3 \mathrm{~s}$ level $\left(b_{3}\right)$. As expected, all three coefficients become very close to 1 in the deep layers, and reach values below 0.5 in surface layers, as found by Carlsson et al. (1994). The adopted logarithmic abundance of $\mathrm{Li}$ is 2.2 , on the scale $\log (\mathrm{nH})=12.0$.

$$
U=\sum_{i} b_{i} g_{i} \exp \left(-\chi_{i} / k T\right)
$$

where the $g_{i}$ and $\chi_{i}$ are the statistical weight and the excitation energy of level $i$, respectively .

\section{Results and discussion}

We have first tested our program on a Kurucz ATLAS 9 1D solar model to see whether the $b_{i}$ had the expected behavior, already computed by Carlsson et al. (1994). Fig. 2 shows the depth-dependence of the first 3 departure coefficients, applying to levels $2 \mathrm{~s}, 2 \mathrm{p}$ and $3 \mathrm{~s}$, respectively.

Next, we have computed the equivalent width of the Li I resonance line for the Kurucz 1D solar model and for two 2D solar snapshots, still for the logarithmic Li abundance 2.2. Fig. 3 shows the variation of the equivalent width 

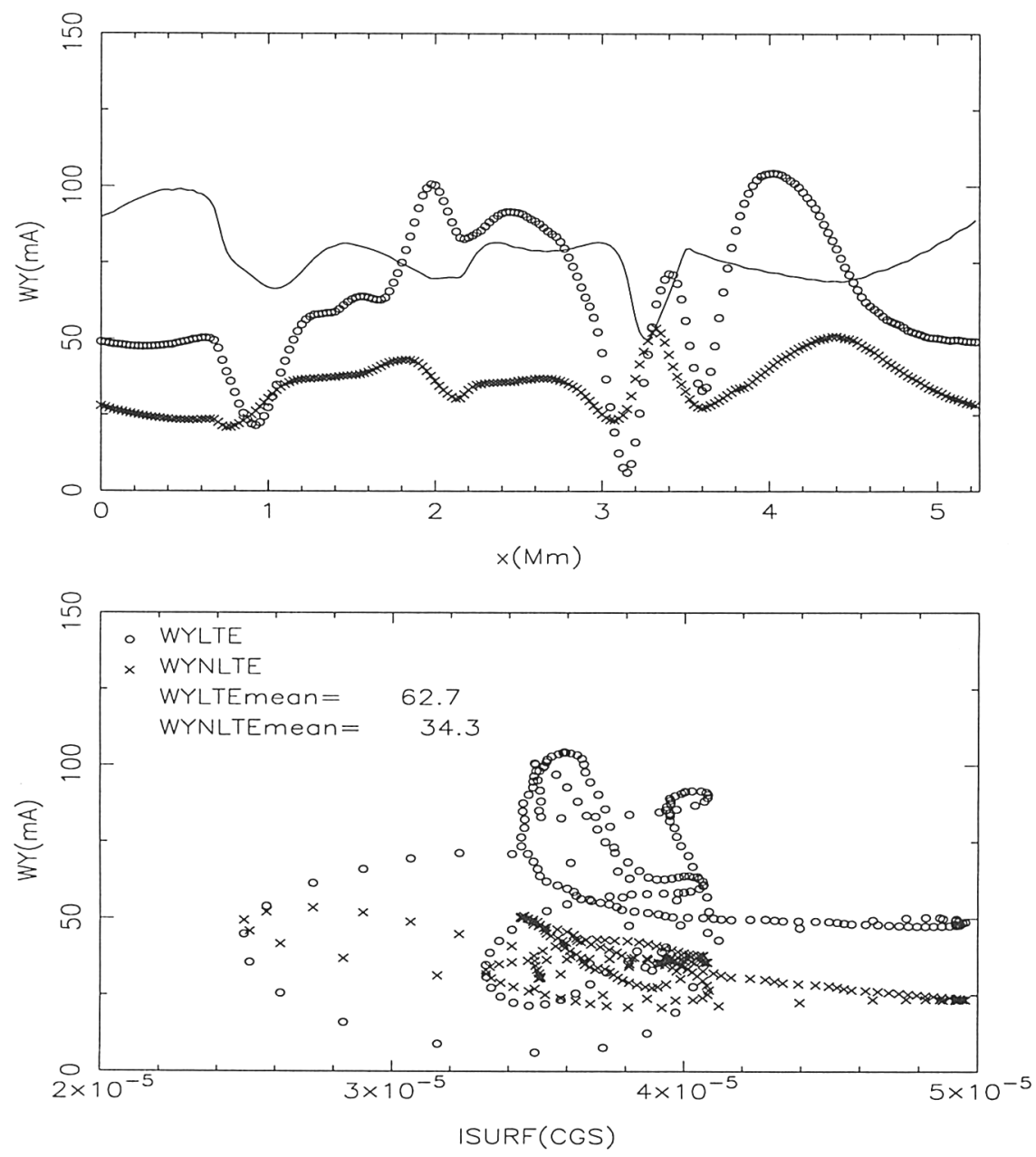

Figure 3. Top: LTE (circles) and NLTE (crosses) equivalent widths of the $\mathrm{Li}$ I resonance line as a function of horizontal position $x$, for a representative snapshot from a $2 \mathrm{D}$ simulation of solar granulation. The thin line gives the continuum intensity at $\lambda 670.8 \mathrm{~nm}$ in arbitrary units. Bottom: Same equivalent widths as a function of continuum intensity ISURF. All data derived from vertical rays $(\mu=1)$. 
Table 1. LTE and NLTE mean equivalent width $\langle W\rangle[\mathrm{m} \AA]$ of the Li I $\lambda 670.8$ line for 2 different snapshots from a $2 \mathrm{D}$ hydrodynamical simulation of solar surface convection, obtained from the horizontally averaged flux spectrum. For comparison, the equivalent widths resulting for a 1D ATLAS9 reference model, computed with the same line formation code, are given in the first row. The results from Kieslman (1997) given in the last rows have been rescaled to the same Li abundance of 2.2 ; they refer to intensity $(\mu=1)$.

\begin{tabular}{ccc}
\hline 1D model or RHD snapshot & $\langle W\rangle$ (LTE) & $\langle W\rangle$ (NLTE) \\
\hline 1D Kurucz $(\alpha=0.5)$ & 43 & 32 \\
2D, L71D09-605 & 59 & 35 \\
2D, L71D09-625 & 71 & 36 \\
\hline Kiselman 1D (OSMARCS) & 44 & 42 \\
Kiselman 3D & 52 & 37 \\
\hline \hline
\end{tabular}

$W$ (for the intensity normal to the surface) of the Li I $670.8 \mathrm{~nm}$ line over the simulated granulation pattern, both as a function of the horizontal position $x$ (top) and as a function of the continuum intensity $I_{c}$ (bottom) for one particular snapshot. Note the wide variation of $W$ computed in LTE, compared to the much more limited excursion of $W$ computed in NLTE. The mean equivalent widths for the flux spectrum integrated over the full length of the sample are given in Table 1 for the two solar snapshots and for the $1 \mathrm{D}$ reference model having the same effective temperature, gravity and (solar) metallicity. In each case, the line is computed in LTE as well as in NLTE. We note that, in NLTE, the results for the 2D snapshots do not differ significantly from the 1D case.

Finally, we have carried out a similar procedure for our metal-poor stellar example, again computing the equivalent width of the $\mathrm{Li}$ I resonance line for a Kurucz 1D model and for five snapshots from our 2D hydrodynamical simulation; as before, a $\mathrm{Li}$ abundance of 2.2 was adopted. Fig. 4 shows the variation of the equivalent width $W$ of the Li I resonance line over the stellar granulation pattern for the typical snapshot displayed in Fig. 1. The difference between LTE and NLTE is even more pronounced than in the solar case, the NLTE correlation between $W$ and $I_{c}$ being much tighter and of opposite sign compared to LTE.

The mean equivalent widths derived from the horizontally averaged flux spectrum are listed in Table 2 for the 5 snapshots and for the Kurucz 1D reference model. In LTE, the 1D/2D difference is huge (granulation abundance correction $\approx-0.45 \mathrm{dex}$ ). But remarkably, the 2D NLTE line strengths show very little dispersion and do not indicate any significant offset with respect to the 1D case. An obvious conclusion from these results is that the 2D LTE computations are way off, strongly underestimating the Li abundance. In NLTE, the error introduced by representing the inhomogeneous stellar atmosphere by a fluxconstant 1D Kurucz model appears to be almost negligible.

The mechanism behind the spatial variation of the line strength is clearly identified on Figs 1 and 4: hot granules produce at the same time a steeper temperature gradient and a lower temperature in the line formation region. In LTE, the latter leads to an overpopulation of the lower level of the transition 

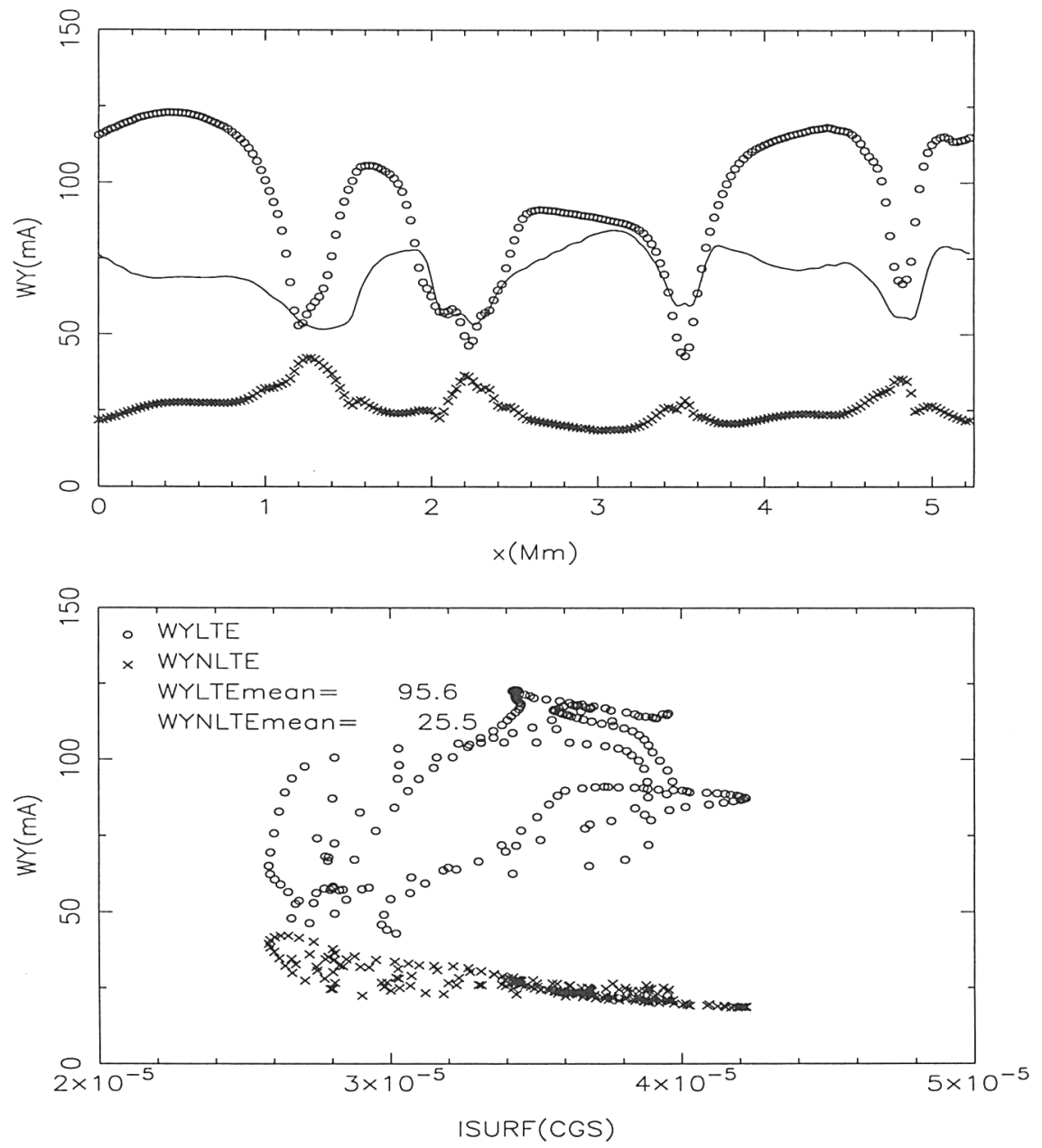

Figure 4. Same plots as Fig. 3, but for the snapshot from the metalpoor star simulation shown in Fig. 1

due to a shift of the ionization equilibrium towards neutral particles (Saha equation). Since both effects enhance the equivalent width of the line, the correlation between continuum intensity and LTE line strength is clearly positive (there is considerable dispersion around the mean $W\left(I_{c}\right)$ relation, however, due to the presence of inclined thermal inhomogeneities). This result is inverse of what is expected for a set of hot and cool radiative equilibrium atmospheres lined up side by side: here the line would weaken in the hot atmosphere, because the population of the lower level is the dominant factor. It is clear that the reasoning of Kurucz fails, essentially because the actual vertical stratification of hot and cold regions has little to do with the stratification of hot and cool radiative equilibrium atmospheres. 
Table 2. Same as Table 1, but for 5 different snapshots from a 2D hydrodynamical simulation of surface convection in a metal-poor star $([M / H]=-2)$ with solar effective temperature and gravity. For comparison, the results for the corresponding 1D ATLAS9 model, computed with the same line formation code, are given in the first row.

\begin{tabular}{ccc}
\hline 1D model or RHD snapshot & $\langle W\rangle(\mathrm{LTE})$ & $\langle W\rangle$ (NLTE) \\
\hline 1D Kurucz $(\alpha=0.5)$ & 38.4 & 26.1 \\
T57G44N7-43108 & 116.0 & 27.7 \\
T57G44N7-69390 & 115.4 & 27.1 \\
T57G44N7-83752 & 112.7 & 27.3 \\
T57G44N7-87007 & 110.9 & 27.7 \\
T57G44N7-97644 & 108.2 & 26.5 \\
\hline \hline
\end{tabular}

A positive $W-I_{c}$ correlation alone is not sufficient to explain the LTE result that the mean equivalent width is much larger in 2D than in 1D: as long as the fluctuations of the line strength with temperature remain in the linear domain, the mean equivalent width is not affected by the temperature fluctuations. But as the population of the ground level $N_{0}$ of Li I varies exponentially with $\mathrm{T}$, a nonlinearity sets in: symmetrical temperature fluctuations produce asymmetrical population variations. Assume that $\mathrm{Li}$ is almost completely ionized and consider only the exponential factor of the Saha equation for simplicity. Then

$$
N_{0}(T)=N_{0}\left(T_{0}\right) \exp (+\chi / k T)=N_{0}\left(T_{0}\right) *\left\{1-q s+q s^{2}+\frac{1}{2} q^{2} s^{2}+\ldots\right\}
$$

where $\chi$ is the ionization potential and we have defined $q \equiv \chi / k T_{0}$ and $s \equiv(T-$ $\left.T_{0}\right) / T_{0}, T_{0}$ being the mean temperature. Taking the horizontal average assuming symmetrical temperature variations $(\langle s\rangle=0)$, we obtain $\langle N\rangle \approx N_{0}\left(T_{0}\right) *(1+$ $0.5 q^{2}\left\langle s^{2}\right\rangle\left(\sqrt{\left\langle s^{2}\right\rangle} \leq 0.04 ; q \approx 12\right)$. So the mean of $W$ is biased towards larger equivalent widths in an inhomogeneous atmosphere. This explains part of the $1 \mathrm{D} / 2 \mathrm{D}$ difference found in our numerical computations. The main contribution, however, is attributed to the lower mean photospheric temperature in the 2D model as a result of adiabatic cooling due to overshooting: $T_{0}(2 D)<T(1 D)$.

In NLTE, the local temperature plays little role. Rather, the radiation field is the dominant factor. Hence, photoionization overionizes the lower level over a hot granule, and the equivalent width becomes smaller. As a result, NLTE equivalent widths are anti-correlated with the continuum surface brightness. The variation of $W$ is much smaller than in LTE, because the angle-averaged radiation field depends only weakly on horizontal position at the height of line formation.

Finally, we would like to mention the work by M. Asplund, who has also presented his NLTE line formation calculations for Li I on this symposium. His results are based on the 3D snapshots that he had used previously for the LTE investigation of the subgiants HD 140283 and HD 84937. He reached, on this completely independent set of hydrodynamical models, and with a different NLTE code, the same conclusions as we did: NLTE line formation in multidimensional models is quite different from the LTE case, in the way that the NLTE 
multi-dimensional $\mathrm{Li}$ abundance is much closer to the abundance derived from classical flux-constant 1D models. A detailed comparison of our results shows that the remaining differences can be traced to the use of a $\mathrm{Li} \mathrm{I}$ atom with 5 levels in our case, and with 20 levels in the case of Asplund et al. (this volume). Another difference is that the temperature inhomogeneities are somewhat enhanced in 2D models with respect of what occurs in $3 \mathrm{D}$ models, leading to correspondingly larger LTE granulation abundance corrections. But this is only a minor point. Certainly, the two groups agree that LTE Li abundance determinations relying on multidimensional hydrodynamical simulations of convection in metal-poor dwarfs are highly misleading.

\section{Conclusions}

1. The statement of Kurucz (1995) that abundances of lithium derived from standard 1D models of metal-poor stellar atmospheres is too small by a factor of 10 is not supported by actual multidimensional NLTE computations. Even the sign of the correction is doubtful, and the error is well below 0.1 dex, both according to our investigation and the one presented by $\mathrm{M}$. Asplund on this symposium.

2. LTE abundance determinations based on inhomogeneous atmospheres are strongly discouraged. They produce large "granulation abundance corrections" due to non-linear effects in the direction opposite to Kurucz' prediction, but the actual NLTE line formation mechanism couples the population of the atomic levels more closely to the mean radiation field than to the local temperature.

3. The combination of multidimensional models with NLTE line formation for the $\mathrm{Li}$ I $\lambda 670.8 \mathrm{~nm}$ resonance line leads to the same lithium abundance as that derived from NLTE analysis with flux-constant 1D models, abundance differences being less than 0.1 dex. However, this result must not be hastily generalized to other atoms with a different atomic structure.

4. An obvious future improvement is to extend the NLTE analysis to the continuum, which has be assumed here to be in LTE. We plan to do that in the near future. If it turns out that the $\mathrm{H}^{-}$ion is affected by NLTE, this will raise a new question: should such effects be included already in the radiation hydrodynamics code, which determines the amplitude of the thermodynamical fluctuations?

\section{References}

Asplund, M., Nordlund, A, Trampedach, R., Stein, R.F. 1999, A\&A, 346, L17

Carlsson, M., Rutten, R.J., Bruls, J.H.M.J., Shchukina, N.G. 1994, A\&A, 288, 860

Freytag, B., Ludwig, H.-G., Steffen, M. 1996, A\&A, 313, 497

Feautrier. P. 1964, C.R. Acad. Sci. Paris, 258, 3189

Gadun A.S., Pavlenko, Ya. V. 1997, A\&A, 324, 281 
Kiselman, D. 1997, ApJ, 489, L107

Kiselman, D. 1998, A\&A, 333, 732

Kurucz, R.L. 1995, ApJ, 452, 102

Ludwig, H.-G., Jordan, S., Steffen, M. 1994, A\&A, 284, 105

Mihalas, D. 1970 Stellar Atmospheres W. H. Freeman \& Co.

Pinsonneault, M.H., Deliyannis, C. P., Demarque, P. 1992, ApJS, 78, 179

Stein, R.F., Nordlund, §̊, 1998, ApJ, 499, 914

Uitenbroek, H. 1998, ApJ, 498, 427 